\title{
Comparison of the expression of human leukocyte antigen (HLA)-G and HLA-E in women with normal pregnancy and those with recurrent miscarriage
}

\author{
A Bhalla, P R Stone, H S Liddell, A Zanderigo and L W Chamley \\ Department of Obstetrics and Gynaecology, Room 3241, Faculty of Medical and Health Sciences, University \\ of Auckland, Private Bag 92019, Auckland, New Zealand \\ Correspondence should be addressed to LW Chamley, Department of Obstetrics and Gynaecology, Rm 3241 FMHS, University \\ of Auckland, Private Bag 92019 Auckland, New Zealand; Email: I.chamley@auckland.ac.nz
}

\begin{abstract}
Recurrent miscarriage affects $\mathbf{1 \%}$ of all couples attempting pregnancy. Immunological factors are postulated to play a role in the aetiology of recurrent miscarriage because the fetus and placenta are immunologically different from the mother. In particular, altered expression of the, non-classical, class I histocompatibility leukocyte antigen (HLA) molecules has been postulated to play a role in the aetiology of recurrent miscarriage as the fetus and placenta are semi-allogenic to the mother. This study was conducted to examine whether altered expression of the non-classical class I HLA molecules, HLA-G and HLA-E, by cells at the maternofetal interface could play a role in the aetiology of recurrent miscarriage. First-trimester placental and decidual biopsies were obtained from 45 women with recurrent miscarriage and 17 gestation-matched normal controls. These biopsies were screened by immunohistochemistry for HLA-G and HLA-E and isotype-matched control antibodies. Staining was analysed by light microscopy and digital image analysis. In both recurrent miscarriage and normal pregnancy, HLA-G was localised to the extravillous trophoblast. There was no difference in the pattern of HLA-G expression between women with recurrent miscarriage and those with normal pregnancies. HLA-E was localised to the syncytiotrophoblast, villous mesenchymal cells, extravillous trophoblast and several decidual cell types, but staining for HLA-E appeared to be confined primarily to the cytoplasm. There was no difference in the pattern of HLA-E expression between women with recurrent miscarriage and those with normal pregnancies.

Reproduction (2006) 131 583-589
\end{abstract}

\section{Introduction}

Recurrent miscarriage (RM) is commonly defined as three or more consecutive first-trimester or two or more consecutive second-trimester pregnancy losses before 20 weeks' gestation. Using this definition, 1-2\% of couples experience RM (Coulam 1991). The risk of a spontaneous miscarriage in clinically identified pregnancy is estimated to be approximately $12-14 \%$ (Edmonds et al. 1982). Consequently, the incidence of three miscarriages by chance alone is in the order of $0.34 \%$ (Stirrat 1990a). This discrepancy implies that, in addition to random causes, some couples are at an increased risk of RM which suggests a specific underlying pathology. Anatomical, infectious, endocrine and genetic factors are all known to be associated with RM. However, the aetiology of RM remains unknown in approximately $50 \%$ of cases (Lee \& Silver 2000).

Potential immunological causes of otherwise unexplained RM have generated considerable interest and controversy. A prominent hypothesis in the area of immune-related RM is that some cases of RM are due to a maternal immune response to the fetus and placenta. This hypothesis holds that the placenta is in effect a foreign tissue (transplant), and that, normally, mechanisms are in place to prevent a maternal immune response to this foreign tissue. It is hypothesised that in some cases of RM these regulatory mechanisms fail, allowing the maternal immune system to respond to fetal antigens. One mechanism that might limit maternal immune responses is immunosuppression of the populations of leukocytes present at the maternofetal interface. During the first trimester of pregnancy the human decidua is rich in leukocytes which make up 10-15\% of all decidual cells (Loke \& King 1995). This leukocyte population is composed of up to $70 \%$ uterine natural killer (NK) cells and 10\% T cells (Loke \& King 1995).

Immunosuppression of these leukocytes is believed to be mediated, in part, by the non-classical class I histocompatibility leukocyte antigen (HLA) molecule, HLA-G. HLA-G 
has been shown to bind to the immunoglobulin-like transcript (ILT)-2 and killer inhibitory receptor (KIR)2DL4 (p49) inhibitory receptors on NK cells and may confer protection to extravillous trophoblasts (EVTs) via these receptors (Allan et al. 1999, Biassoni et al. 1999). Recently, a second non-classical class I HLA molecule, HLA-E, has been shown to be expressed by trophoblasts and HLA-E is also believed to help the fetus to avoid maternal immune surveillance, possibly by interacting with the CD94/NKG2A NK-cell inhibitory receptor (Braud et al. 1998, Lee et al. 1998).

This investigation was undertaken in order to determine whether there was an altered expression of HLA-G or HLA-E at the maternofetal interface in women with RM compared with women with normal pregnancies.

\section{Materials and Methods}

\section{Tissues}

This study was approved by the Auckland Regional Health and Disability Ethics Committee and all tissue samples were obtained with informed consent.

Collection of the specimens has been described previously (Askelund et al. 2004); briefly, decidual biopsies from the implantation site and/or villous placental samples were collected from 45 women attending the RM clinic at the National Women's Hospital by ultrasound- guided curettage as soon as possible after confirming embryonic/fetal demise. This usually occurred within $6 \mathrm{~h}$ of the confirmation of fetal demise (Fig. 1). In all cases this was prior to the onset of vaginal bleeding.

Women attending the RM clinic were extensively investigated and monitored closely. Routine investigations included full blood count, rubella, hepatitis B and syphilis serology; and blood group and antibody screen. In addition, women were screened for antinuclear antibodies and extractable nuclear antigens, anticardiolipin antibodies and lupus anticoagulant. Women were also investigated for activated protein $C$ resistance and if this analysis was abnormal they were screened for Factor $\mathrm{V}$ Leiden mutations. All women had swabs for bacterial vaginosis. Quantitative $\beta$-human chorionic gonadotrophin ( $\beta \mathrm{HCG}$ ) assays were performed three times weekly until 5 weeks

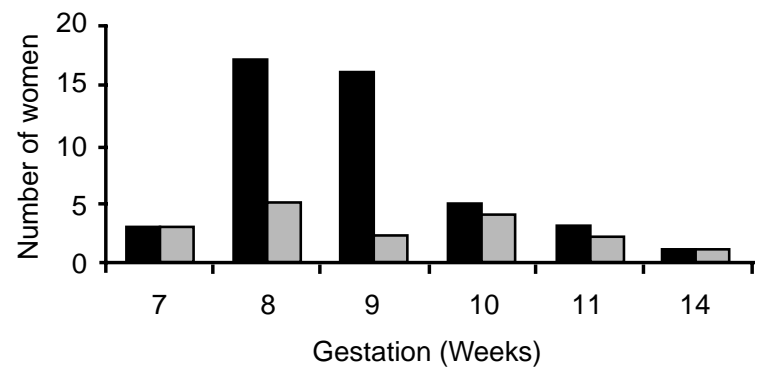

Figure 1 Samples for this study were obtained from a total of 62 women and are presented by gestational age. Women with RM (black bars); women undergoing termination of pregnancy (grey bars). of gestation and then weekly until 9 weeks of gestation. Ultrasound scans were performed weekly from 6 gestational weeks. Consequently, early pregnancy failure was generally predicted by either an abnormal trend in serial $\beta$ HCG measurements or ultrasound findings. As soon as embryonic or fetal demise was confirmed, evacuation of the uterus was arranged. The method of ultrasound-guided biopsy has been described previously (Khong et al. 1987). All products of conception were analysed for fetal karyotype, 15 were of normal karyotype, 5 failed to grow and therefore could not be karyotyped and the remaining pregnancies had abnormal karyotypes. In all cases, gestational age assessment was based on crown rump length measurement by ultrasound. Gestation-matched decidual biopsies and/or villous placental tissues were also collected from 17 women undergoing an elective surgical termination of pregnancy (TOP) (Fig. 1). The gestational age and fetal viability of all pregnancies in this group were confirmed by ultrasound assessment. In no cases in this group was there vaginal bleeding or evidence of embryonic or fetal demise prior to the termination of pregnancy.

All samples were embedded in cryoembedding compound (TBS, Biotek Auckland, New Zealand) and snap frozen in liquid nitrogen-cooled 2-methyl butane then stored at $-80^{\circ} \mathrm{C}$ until required. Serial thin sections $(5 \mu \mathrm{m})$ were cut using a cryostat (Leica CM1900, Germany), fixed for $10 \mathrm{~min}$ in cold acetone, air-dried, then wrapped in aluminum foil and parafilm and stored at $-20^{\circ} \mathrm{C}$ until use.

\section{Immunohistochemistry}

Slides were thawed and non-specific binding sites were blocked by the addition of $100 \mu \mathrm{l} 10 \%$ normal goat serum (blocking solution) (Life Technologies, Auckland, New Zealand), diluted in PBS, pH 7.4, containing $0.05 \%$ Tween 20 (PBS-Tween) (Serva, Heidelberg, Germany) for $10 \mathrm{~min}$. The slides were then washed three times in PBS-Tween for $2 \mathrm{~min}$. Following this, the slides were incubated with $50 \mu \mathrm{l}$ of the primary antibody in $10 \%$ normal goat serum in PBS-Tween for $1 \mathrm{~h}$ at room temperature. The HLA-G antibody, MEM-G/9 (Abcam, Cambridge, UK) was used at a dilution of 1:200 and the HLA-E antibody, MEM-E/02 (Abcam), was used at a dilution of 1:300. Both MEM-G/9 and MEM-E/02 are IgG1 isotype antibodies. The slides were then washed three times in PBS-Tween for 2 min per wash and incubated with $50 \mu \mathrm{l} 5 \% \mathrm{H}_{2} \mathrm{O}_{2}$ in methanol for $5 \mathrm{~min}$ to quench endogenous peroxidase activity. The slides were then washed three times in PBS-Tween (2 min/wash) and incubated with $50 \mu \mathrm{l}$ biotinylated (goat) secondary antibody (broad-spectrum staining kit; Zymed, San Fransisco, CA, USA) for $10 \mathrm{~min}$ at room temperature. Following this the slides were washed again in PBS-Tween and incubated with $50 \mu$ l streptavidinconjugated horse radish peroxidase (Zymed) for $10 \mathrm{~min}$ at room temperature. The slides were washed a further three times with PBS-Tween (2 min/wash) and incubated with 
$50 \mu \mathrm{l}$ of the chromagen 3-amino-9-ethylcabazole (AEC) (Dako, MedBio, Christchurch, New Zealand) for $20 \mathrm{~min}$ at room temperature. Finally, the slides were washed for $2 \mathrm{~min}$ in deionised water and counterstained with Gills No. 2 haematoxylin (Amber Scientific, Australia) for $30 \mathrm{~s}$. The slides were then washed in tap water and coverslips (BioLab Scientific, Auckland, New Zealand) mounted using Aquamount (BHD, Palmerston North, New Zealand). Additional slides were stained with IgG1 isotype antibodies reactive with cytokeratin, CD45, CD3, CD56 (Dako), CD57 (IgM, Novo Castra, Newcastle upon Tyne, UK) and CD14 (IgG2b isotype, this laboratory) using the same method. The semi-serial sections were used as isotypematched controls and also to assist in the identification of cell types within the tissues.

\section{Analysis of HLA-G and HLA-E immunostaining}

Sections were analysed for HLA-G and HLA-E staining using a light microscope (Nikon ECLIPSE E400, Japan) and photomicrographs were taken using a Nikon Coolpix 990 digital camera (Nikon). EVTs were identified based on cytokeratin staining in semi-serial sections. Cells immunostained for HLA-E were identified based on morphology and staining patterns seen in semi-serial sections stained with HLA-G, cytokeratin, CD45 and CD14.

The HLA-G and HLA-E staining was analysed descriptively. HLA-G staining in EVTs was then analysed semiquantitatively by grading the intensity of HLA-G expression on a scale of 0 to 3 (where: $0=$ no staining; $1=$ light staining intensity; $2=$ medium staining intensity; $3=$ strong staining intensity). Statistical significance of differences between groups was assessed using the Wilcoxon rank sum test. All differences were considered to be statistically significant when the $P$ value was $\leq 0.05$.

HLA-E expression was further analysed by determining the percentage of those samples in which specific cell types stained for HLA-E. Whether differences were statistically significant was assessed using Fisher's exact test for percentages. All differences were considered to be statistically significant when the $P$ value was $\leq 0.05$.

\section{Results}

\section{HLA-G expression}

In the trophoblast columns HLA-G immunostaining was localised to EVTs from women with normal pregnancies and those with RM while there was no staining of any cell type in the villi. Furthermore, the staining intensity of HLA$\mathrm{G}$ increased as EVTs migrated further away from the villi (Fig. 2). There were two samples from women with normal pregnancies, as well as two samples from women with RM, that contained trophoblast columns that did not stain for HLA-G (Fig. 2). There was strong immunostaining for HLA$G$ in EVTs that had invaded the decidua, including endovascular trophoblast (Fig. 2). There were no obvious differences in the expression pattern of HLA-G between women with RM and those with normal pregnancies.

Semi-quantiative analysis revealed that the mean HLA-G staining intensity in the proximal trophoblast columns from women with RM (2.1) was not significantly different $(P=0.051)$ from that in women with normal pregnancies (1.55). Likewise, there was no significant difference in the staining intensity of HLA-G in decidual EVTs between women with RM and those with normal pregnancies $(P=0.072)$.

We also compared the staining intensity of HLA-G, in a subgroup of women with RM whose products of conception were karyotypically normal (2.31), with the HLA-G staining intensity in the women with normal pregnancies (1.55) but found no significant difference $(P=0.069)$.

\section{HLA-E expression}

In general there was no staining for HLA-E in the villi. However, very rarely the cytoplasm of syncytiotrophoblast in sporadic villi stained strongly positive for HLA-E (Fig. 3F). EVTs in some, but not all, proximal columns and placental septae stained strongly for HLA-E (Fig. 3B).

HLA-E immunostaining was localised to some interstitial trophoblast in the decidual stroma. Strong HLA-E immunostaining was found on large decidualised stromal cells and uterine epithelium (Fig. 3C), uterine endothelial cells also stained. In all of these three cell types HLA-E appeared to be limited to the cytoplasm but a few large decidualised stromal cells exhibited a rim pattern of staining. Other decidual stromal cells also stained positive for HLA-E (Fig. 3C) and based on their morphology and the staining patterns observed in semi-serial sections stained with CD56, CD3 or CD14, these cells were thought to be leucocytes.

Semi-quantitative analysis revealed that there was no significant difference in the expression of HLA-E by EVTs in the proximal columns $(68 \%$ vs $58 \% ; P=0.072)$ or in the decidua $(33 \%$ vs $72 \% ; P=0.10)$ between women with RM and those with normal pregnancies respectively.

Comparison of the expression of HLA-E and HLA-G revealed that $35 \%$ of the samples contained EVTs in the proximal trophoblast columns that expressed HLA-G but not HLA-E (Fig. 2A and B). Furthermore, one specimen contained EVTs in a proximal column that were immunostained for HLA-E in the absence of HLA-G immunostaining (Fig. 3A and B).

\section{Discussion}

HLA-G is a, non-classical, class I HLA molecule, exhibiting low polymorphism that is postulated to mediate, in part, maternal tolerance to the semi-allogenic fetus by acting as an invariant 'fetal' signal (Loke \& King 1997, Stirrat 1990b). HLA-G may perform this function by interacting with the NK-cell inhibitory receptors ILT-2 and the KIR2DL4 (p49) receptor found on uterine NK cells (Biassoni et al. 1999), 

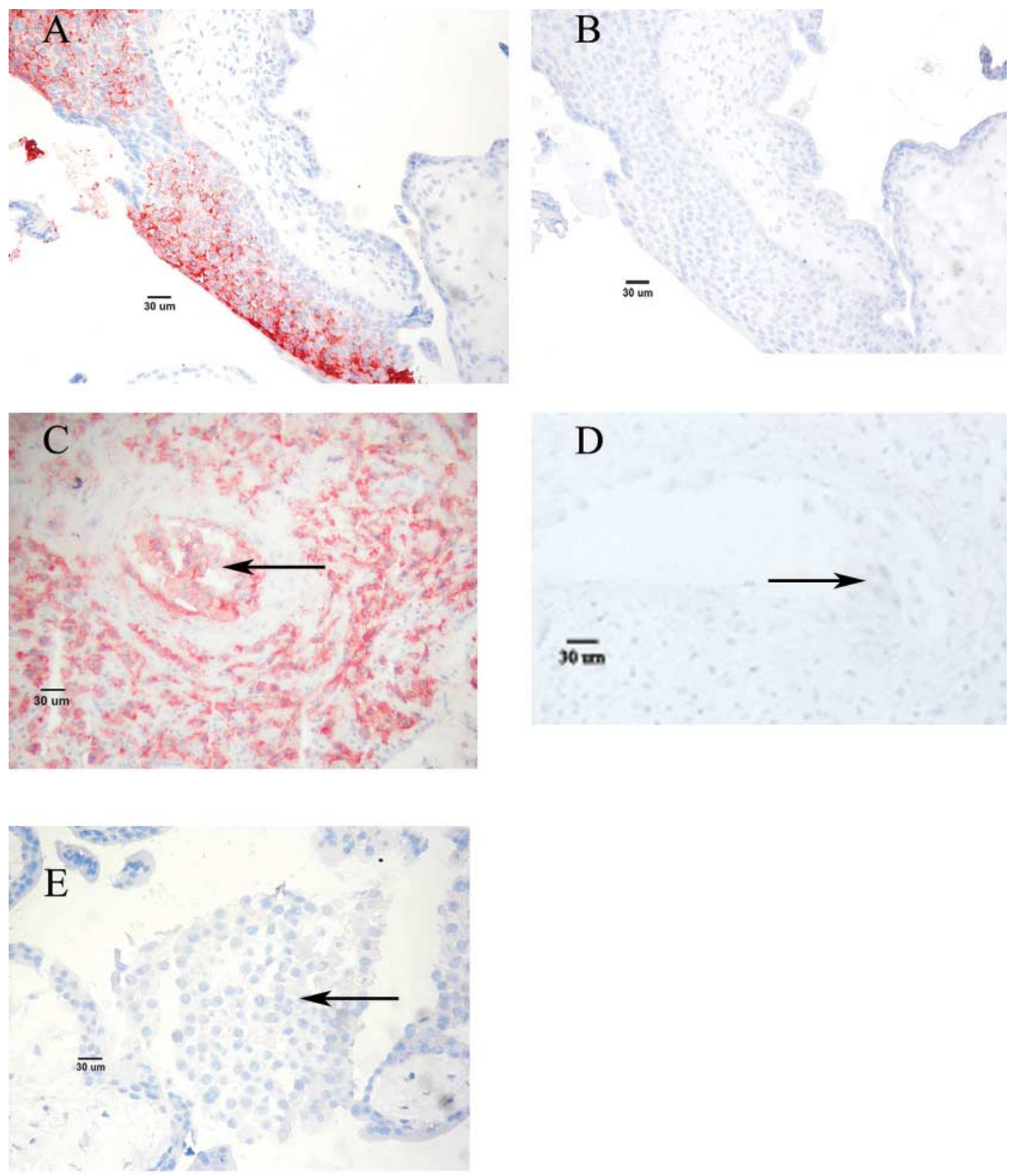

Figure 2 Photomicrographs depicting the immunohistochemical localisation of HLA-G and HLA-E in the placenta and decidua. (A) Gradient in the staining of HLA-G expression in a proximal trophoblast column. (B) Lack of expression of HLA-E in a section semi-serial to panel A. (C) Localisation of HLA-G in endovascular trophoblast (arrow) and decidual EVTs. (D) Section semi-serial to panel C stained with negative control anti-CD57 (arrow indicates endovascular trophoblast). (E) EVTs in a proximal column showing no HLA-G staining (arrow).

as well as with the T-cell receptor, to inhibit NK-mediated cytolysis and allogenic T-cell responses respectively (Riteau et al. 1999). Further evidence for the role of HLA-G in mediating maternofetal tolerance is that it is only expressed by EVTs (Loke \& King 1995). EVTs are the only fetal cells that invade the decidua thereby coming into direct contact with cells of the maternal decidual immune system (Kovats et al. 1990, Wei \& Orr 1990).

The results of this study are in accordance with previous studies showing that the membrane-bound HLA-G isoform is only expressed by EVTs. In addition, there is a gradient of staining, such that as EVTs migrate further away from the villi and into the decidua, they increase their expression of HLA-G. Most EVTs express HLA-G and this expression appeared consistent with localisation to the cell membrane. EVTs that had invaded the decidua strongly expressed HLA-G in both women with normal pregnancies and those with RM. This finding excludes the possibility that RM could be explained solely by the lack of HLA-G expression by EVTs.

Semi-quantitative analysis revealed that there was no statistically significant difference in the mean intensity of 

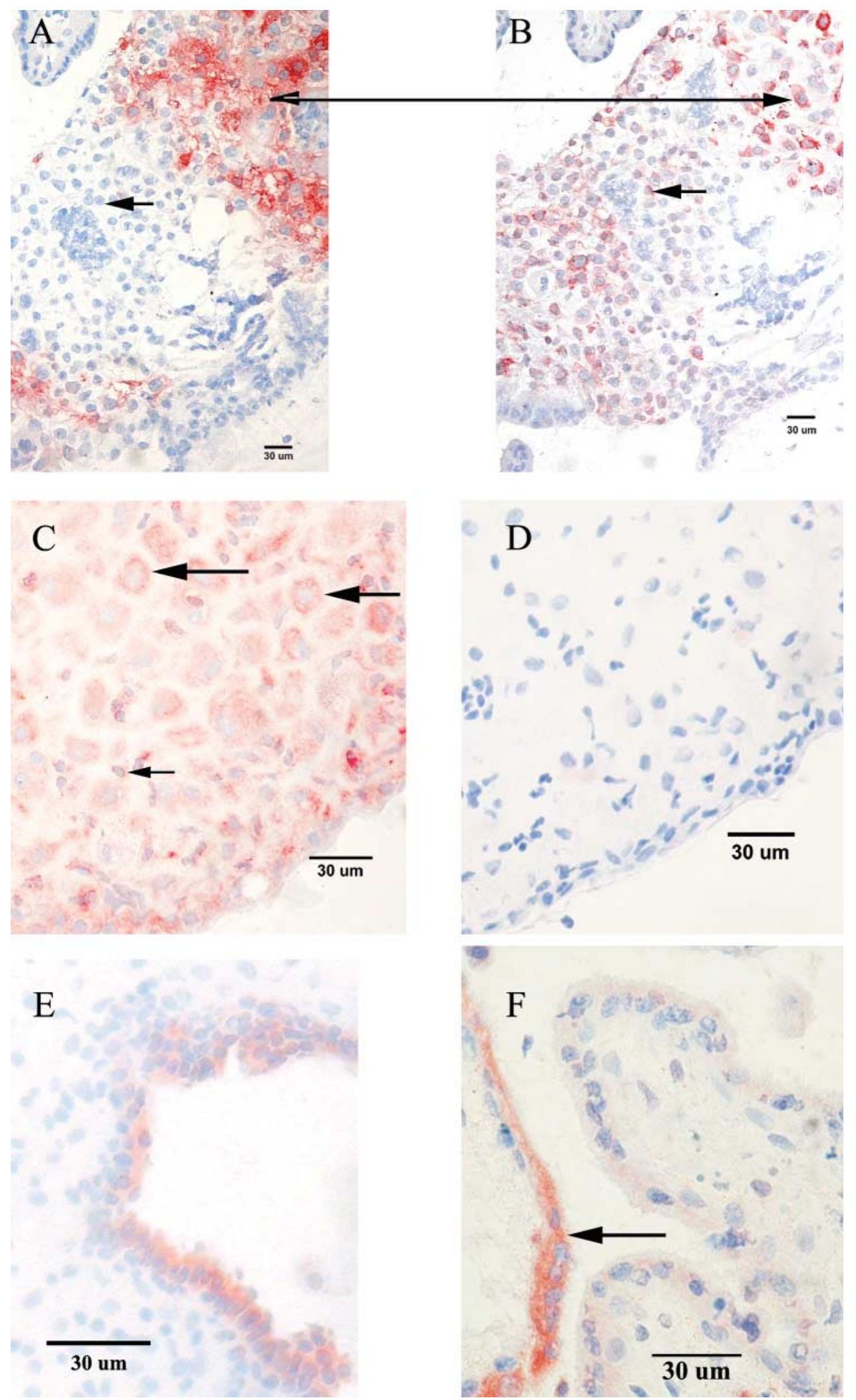

Figure 3 Photomicrographs depicting the immunohistochemial localisation of HLA-E in the placenta and decidua. Semi-serial sections through a proximal trophoblast column were stained for (A) HLA-G and (B) HLA-E; while some trophoblasts in this column expressed both HLA-G and HLA-E (double-ended arrow) other trophoblasts expressed HLA-E but not HLA-G (arrows). (C) HLA-E was also localised to large decidual stromal cells (large arrows) and decidual leucocytes (small arrow). (D) Section semi-serial to panel C stained with an isotype-matched control antibody reactive with CD56. (E) HLA-E expression in glandular epithelium; (F) HLA-E was localised to sporadic areas of syncytiotrophoblast (arrow). 
HLA-G staining by EVTs between women with normal pregnancies or with RM. This result is consistent with one study which also found no difference in the expression of HLA-G between women with RM and with normal pregnancies (Patel et al. 2003), but is contrary to the findings of Emmer et al. (2002) who observed a lower intensity of HLA-G staining of decidual EVTs from women with RM compared with those from women with normal pregnancies. However, Emmer et al. (2002) examined samples from only two normal pregnancies and nine women with RM; such a sample size is likely to be inadequate to allow valid conclusions to be drawn. Thus, results from this study suggest that RM is not caused by a lack of HLA-G expression by EVTs. This finding is supported by a previous report of a woman who was homozygous for the HLA-G null allele (Ober et al. 1998), who had survived life in utero, as a fetus whose placenta could not express HLA-G, for 9 months. While this study suggests that RM is not associated with a lack of expression of HLA-G protein by EVTs, it remains possible that the HLA-G protein expressed by EVTs in women with RM may be functionally defective.

In this study (using the MEM-G/09 antibody) we did not detect HLA-G on placental endothelial cells or other villous stromal cell types. Other workers have reported that HLA-G is localised to fetal endothelial cells both in the first trimester and at term (Blaschitz et al. 1997, 2001, Dye et al. 2001). However, detection of HLA-G on placental endothelial cells appears to be dependent upon the specific epitope within HLA-G recognised by individual monoclonal antibodies.

EVTs have been shown to express the, non-classical, class I HLA-E protein (Wei \& Orr 1990, King et al. 2000, Ishitani et al. 2003). HLA-E may also be perceived as a 'self' signal by maternal NK cells expressing the inhibitory receptor CD94/NKG2A, thus collaborating with HLA-G to prevent cytotoxicity (King et al. 2000). Results from this study demonstrate that, in accordance with previous studies, HLA-E is expressed by EVTs. However, in contrast to the findings of Ishitani et al. (2003), villous cytotrophoblasts were not found to express HLA-E and while we did find expression of HLA-E by some syncytiotrophoblasts, this rare staining was not typical. We also found that in the decidua, HLA-E is expressed by large decidualised stromal cells, glandular epithelium, uterine endothelial cells and by decidual leukocytes.

Cell surface expression of any class I HLA molecule requires the formation of a trimolecular complex consisting of the HLA class I heavy chain, $\beta$ microglobulin, and the peptide antigen that will be presented to T cells. In the case of HLA-E, these peptides are derived from the N-terminal signal sequences of some HLA class I molecules, especially that of HLA-G but also HLA-C (Braud et al. 1997, Lee et al. 1998, Navarro et al. 1999). Binding of leader peptides from MHC class I molecules stabilises the HLA-E protein and allows it to migrate to the cell surface where it can interact with the NK-cell inhibitory receptor CD94/NKG2A. In EVTs cell surface expression of HLA-E is thought to be primarily dependent on HLA-G expression, although HLA-C may also be involved. Syncytiotrophoblast and villous mesenchymal cells do not express the transmembrane form of HLA-G but do express, in a limited fashion, HLA-E. This suggests that HLA-E expression might be dependent on the expression of the soluble form of HLA-G1 which is reportedly expressed by syncytiotrophoblasts (Ishitani et al. 2003). Others have shown that the additional splice variants of HLA-G can not support the cell surface expression of HLA-E (Ulbrecht et al. 2004).

Ishitani et al. (2003) reported that all cells expressing HLA-G expressed HLA-E. While we found that all cell types expressing HLA-G also expressed HLA-E, we did find that a number of individual cells, in particular decidual EVTs, expressed HLA-G but not HLA-E. The fact that some cells did not coexpress HLA-E with HLA-G suggests that HLA-G expression is not sufficient to ensure expression of HLA-E in EVTs. Others have recently suggested that a similar situation occurs in tumour cell lines, where the presence of class I HLA-A or HLA-C proteins that should allow HLA-E expression does not necessarily lead to expression of HLA-E on the cell surface (Palmisano et al. 2005).

In an immunohistochemical study such as ours it is not possible to determine whether immunoreactive proteins are expressed at the cell surface or not. However, HLA-E reactivity in most cell types, including EVTs, appeared to be limited to the cytoplasm and did not appear to be associated with the cell membrane. This suggests that the HLA-E we detected in many cells was not expressed on the cell surface. This leads us to question the functional significance of the apparent expression of HLA-E at the maternofetal interface.

In agreement with others (Wei \& Orr 1990, Blaschitz et al. 2001, Ishitani et al. 2003) we found a number of cell types that appeared to express HLA-E, but the staining for HLA-E was often inconsistent. It is important to stress this point because, while HLA-E is expressed by a variety of cell types in the placenta and decidua, not all of the cells of a given type express HLA-E. For example, some small regions of syncytiotrophoblast express HLA-E while the majority of the syncytiotrophoblast does not. The reason why the staining for HLA-E is not uniform remains unclear and leads us again to question the functional significance of HLA-E expression. In contrast to our results, others, using different antibodies, have reported no expression of HLA-E in any trophoblast cell population (Blaschitz et al. 2001); however, this conflict could be explained by the epitope specificity of the different antibodies.

One limitation of this and similar studies is that the small sample size may mean that the study was underpowered to detect important differences in the expression of HLA molecules between RM and normal pregnancy. However, the fact that the two larger studies conducted to date, ours and that of Patel et al. (2003), both found no 
difference in the expression of HLA-G between RM and normal pregnancy suggests that this is not the case.

In conclusion, we have shown that there is no significant difference in the expression patterns of HLA-G or HLA-E at the maternofetal interface in women with RM compared with women with normal pregnancies. We have also shown that expression of HLA-G is not sufficient to ensure co-expression of HLA-E and we question the functional significance of HLA-E at the maternofetal interface.

\section{Acknowledgements}

We thank Ms N Fernando for her excellent technical assistance. We wish to thank the staff of the Epsom Day Unit, Green Lane Hospital and Dr A MacKintosh for their assistance in obtaining access to patients. This work was funded by grants from the Evelyn Bond Trust, The Health Research Council of New Zealand, The New Zealand Lotteries Board (Health) and the University of Auckland Staff Research Fund. The authors declare that there is no conflict of interest that would prejudice the impartiality of this scientific work.

\section{References}

Allan D, Colonna M, Lanier L, Churakova T, Abrams J, Ellis S, McMichael A \& Braud V 1999 Tetrameric complexes of human histocompatibility leukocyte antigen HLA-G bind to peripheral blood myelomonocytic cells. Journal of Experimental Medicine $\mathbf{1 8 9}$ 1149-1156.

Askelund K, Liddell H, Zanderigo A, Fernando N, Khong T, Stone P \& Chamley L 2004 CD83 + dendritic cells in the decidua of women with recurrent miscarriage and normal pregnancy. Placenta 25 140-145.

Biassoni R, Bottino C, Millo R, Moretta L \& Moretta A 1999 Natural killer cell-mediated recognition of human trophoblast. Seminars in Cancer Biology 9 13-18.

Blaschitz A, Lenfant F, Mallet V, Hartmann M, Bensussan A, Geraghty D, Le Bouteiller P \& Dohr G 1997 Endothelial cells in chorionic fetal vessels of first trimester placenta express HLA-G. European Journal of Immunology 27 3380-3388.

Blaschitz A, Hutter H \& Dohr G 2001 HLA Class I protein expression in the human placenta. Early Pregnancy 5 67-69.

Braud V, Jones E \& McMichael A 1997 The human major histocompatibility complex class Ib molecule HLA-E binds signal sequence-derived peptides with primary anchor residues at positions 2 and 9. European Journal of Immunology 27 1164-1169.

Braud V, Allan D, O'Callaghan C, Soderstrom K, D'Andrea A, Ogg G, Lazetic S, Young N, Bell J, Phillips J, Lanier L \& McMichael A 1998 HLA-E binds to natural killer cell receptors CD94/NKG2A, B and C. Nature 391 795-799.

Coulam C 1991 Epidemiology of recurrent spontaneous abortion. American Journal of Reproductive Immunology 26 23-27.

Dye J, Jablenska R, Donnelly J, Lawrence L, Leach L, Clark P \& Firth J 2001 Phenotype of the endothelium in the human term placenta. Placenta 22 32-43.

Edmonds D, Lindsay K, Miller J, Williamson E \& Wood P 1982 Early embryonic mortality in women. Fertility and Sterility 38 447-453.

Emmer P, Steegers E, Kerstens H, Bulten J, Nelen W, Boer K \& Joosten I 2002 Altered phenotype of HLA-G expressing trophoblast and decidual natural killer cells in pathological pregnancies. Human Reproduction 17 1072-1080.

Ishitani A, Sageshima N, Lee N, Dorofeeva N, Hatake K, Marquardt H \& Geraghty D 2003 Protein expression and peptide binding suggest unique and interacting functional roles for HLA-E, F, and G in maternal-placental immune recognition. Journal of Immunology $\mathbf{1 7 1}$ 1376-1384.

Khong T, Liddell H \& Robertson W 1987 Defective haemochorial placentation as a cause of miscarriage: a preliminary study. British Journal of Obstetrics and Gynaecology 94 449-455.

King A, Allan D, Bowen $M$, Powis S, Joseph S, Verma S, Hiby S, McMichael A, Loke Y \& Braud V 2000 HLA-E is expressed on trophoblast and interacts with CD94/NKG2 receptors on decidual NK cells. European Journal Immunology 30 1623-1631.

Kovats S, Main E, Librach C, Stubblebine M, Fisher S \& DeMars R 1990 A class I antigen, HLA-G, expressed in human trophoblasts. Science 248 220-223.

Lee N, Llano M, Carretero M, Ishitani A, Navarro F, Lopez-Botet M \& Gherarty D 1998 HLA-E is a major ligand for the natural killer inhibitory receptor CD94:NKG2A. PNAS 95 5199-5204.

Lee R \& Silver R 2000 Pregnancy loss: summary and clinical recommendations. Seminars in Reproductive Medicine 18 433-440.

Loke Y \& King A 1995 Human Implantation: Cell Biology and Immunology, Cambridge: Cambridge University Press.

Loke Y \& King A 1997 Immunology of human placental implantation: clinical implications of our current understanding. Molecular Medicine Today 3 153-159.

Navarro $F$, Llano $M$, Bellon $T$, Colonna $M$, Geraghty D \& Lopez-Botet M 1999 The ILT2(LIR1) and CD94/NKG2A NK cell receptors respectively recognize HLA-G1 and HLA-E molecules co-expressed on target cells. European Journal Immunology 29 $227-283$.

Ober C, Aldrich C, Rosinsky B, Robertson A, Walker M, Willadsen S, Verp M, Geraghty D \& Hunt J 1998 HLA-G1 protein expression is not essential for fetal survival. Placenta 19 127-132.

Palmisano G, Contardi E, Morabito A, Gargaglione V, Ferrara G \& Pistillo M 2005 HLA-E surface expression is independent of the availability of HLA class I signal sequence-derived peptides in human tumor cell lines. Human Immunology 66 1-12.

Patel RN, Quack KC, Hill JA \& Schust DJ 2003 Expression of membrane-bound HLA-G at the maternal-fetal interface is not associated with pregnancy maintenance among patients with idiopathic recurrent pregnancy loss. Molecular Human Reproduction $9551-557$.

Riteau B, Menier C, Khalil-Daher I, Sedlik C, Dausset J, Rouas-Freiss N \& Carosella E 1999 HLA-G inhibits the allogeneic proliferative response. Journal of Reproductive Immunology 43 203-211.

Stirrat G 1990a Recurrent miscarriage I: definition and epidemiology. Lancet 336 673-675.

Stirrat G 1990b Recurrent miscarriage II: clinical associations, causes, and management. Lancet $336728-733$.

Ulbrecht M, Maier S, Hofmeister V, Falk CS, Brooks AG, McMaster MT \& Weiss EH 2004 Truncated HLA-G isoforms are retained in the endoplasmic reticulum and insufficiently provide HLA-E ligands. Human Immunology 65 200-208.

Wei X \& Orr H 1990 Differential expression of HLA-E, HLA-F, and HLA-G transcripts in human tissue. Human Immunology 29 $131-142$.

Received 19 July 2005

First decision 26 August 2005

Revised manuscript received 26 October 2005

Accepted 17 November 2005 